\title{
Comparison of Whole-Blood Glutathione Peroxidase Activity, Levels of Serum Selenium, and Lipid Peroxidation in Subjects from the Fishing and Rural Communities of "Rabo de Peixe" Village, San Miguel Island, The Azores' Archipelago, Portugal
}

M. L. PavÃo, ${ }^{*}, 1$ C. Cordeiro, ${ }^{1}$ A. Costa,${ }^{2}$ J. A. Raposo, ${ }^{2}$ M. C. Santos, ${ }^{3}$ J. NÈVE, ${ }^{5}$ And A. M. VIEGAS-CRESPO ${ }^{4}$

${ }^{1}$ CIRN, University of The Azores, R. Mãe de Deus, 9500-801-Ponta Delgada, Portugal; ' ${ }^{2}$ Centro de Saúde, 9000-Ribeira Grande, Portugal; ${ }^{3} C E B F$, Department of Chemistry and Biochemistry, and ${ }^{4} \mathrm{CBA}$, Department of Zoology, Faculty of Sciences, University of Lisbon, 1749-016 Lisbon, Portugal; and ${ }^{5}$ Institute of Pharmacy, Free University of Brussels, Campus Plaine 205-5, B-1050 Brussels, Belgium

Received May 3, 2002; Revised August 13, 2002; Accepted August 8, 2002

\section{ABSTRACT}

The activity of glutathione peroxidase (GSH-Px), serum selenium (Se), and thiobarbituric acid reactive substances (TBARS) were measured in the whole blood of 148 healthy adults aged 20-60 yr from the fishing and rural communities of "Rabo de Peixe," The Azores, Portugal.

The subjects did not live in the same household and had different socioeconomic profiles and dietary habits. The serum lipid profile and selected life habits were also considered in this study. No significant differences in the activity of GSH-Px were found in the interpopulation or intrapopulation analyses, classified by age or lipid profile.

*Author to whom all correspondence and reprint requests should be addressed. 
An age-dependent GSH-Px increase was noted in the younger male (M) subgroups (20-39 yr). The Se levels were higher in fishers (f) of both genders $(\mathrm{M}, \mathrm{F})$ than in subjects living in the rural (r) environment: $110 \pm 25$

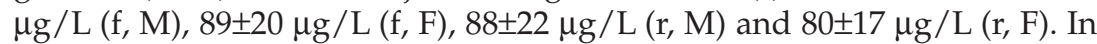
the fishers, but not in the rural population, Se was higher in the males, but it did not show significant variation with age. The levels of TBARS were lower in the $\mathrm{f}$ than in the r male group. The Se level was lower and TBARS higher in the hyperlipemic women in the f group, compared to the corresponding controls.

Our results suggest that the fishers (mainly men) show a better antioxidant status than that of their rural counterparts, due to differences in dietary habits between the study populations and between genders.

Index Entries: Antioxidants; selenium; glutathione peroxidase; TBARS, The Azores.

\section{INTRODUCTION}

The primary enzymatic antioxidant defense of human cells include the four selenium-dependent glutathione peroxidase (GSH-Px) enzymes, all having the ability to catalyze the decomposition of hydrogen peroxide and of various alkyl hydroperoxides at the expense of glutathione. There is increasing evidence that the individual GSH-Px isozymes, which are not equally distributed among tissues and organs, also respond differently in expression and activity to selenium (Se) deprivation and subsequent resupplementation. This suggests the existence of an internal ranking order pointing to specific roles in metabolic regulation played by the different isozymes, beyond their antioxidant potential (1).

In blood, GSH-Px is distributed almost exclusively within the red cells (2), where Se is incorporated as selenocysteine in cytosolic (or classic) glutathione peroxidase (cGSH-Px) during erythropoiesis, with the primary function to protect the organism against oxidative attack. Plasma glutathione peroxidase (pGSH-Px) is a major extracellular, fast-acting hydroperoxide-reducing enzyme in human plasma that accepts other thiol-reducing substrates instead of glutathione $(3,4)$. It is likely that pGSH-Px plays roles in addition to being an antioxidant. For example, it might also regulate the extracellular concentration of hydroperoxides supposed to be relevant to lipid-mediated biosynthesis (5).

Diet also influences blood Se levels and the pro-oxidant and antioxidant balance in the organism. As reported in several epidemiological studies carried out in people with specific life habits or in residents of particular geographic areas, age, gender, medical condition or treatment, and living style are some aspects that determine antioxidant status in human $(2,6)$.

Imbalance between reactive oxygen species (ROS) and the antioxidant cell defenses is known to be relevant in the genesis of several human disorders such as atherosclerosis, diabetes, and inflammatory diseases, among 
others $(7,8)$. Lipid peroxidation, namely of polyunsaturated fatty acids transported by low-density lipoproteins (LDLs), is an important feature of the injury caused by ROS and a major source of secondary cytotoxic products (9).

This work is part of a project aimed at an overview of the oxidant/antioxidant balance in several Portuguese populations with different sociocultural profiles, including urban, rural, and fishing communities. A few small Azorean villages still exhibit unique lifestyles that are linked to specific geographic isolation, economic basis of subsistence, and dietary habits. These studies are important in terms of public health, health prevention, and education issues.

The purpose of this study was to compare the whole-blood GSH-Px activity, serum Se concentration, and thiobarbituric acid reactive substances (TBARS) (as an index of lipid peroxidation) in apparently healthy subjects from the fishing and the rural communities of "Rabo de Peixe," San Miguel Island, The Azores' Archipelago, Portugal. The serum lipid profile of the subjects, as well as age, gender, and some habits like alcohol, drug, and tobacco consumption were also considered.

\section{METHODS}

\section{Study Subjects}

The subjects selected to participate in this study were 72 volunteers (28 males, 44 females) from the fishing community and 76 volunteers (26 males, 50 females) from the rural communities of "Rabo de Peixe," in the Azores. The village is located on the north coast of the island of San Miguel and has about 6650 inhabitants, of which about 1000 are dedicated to fishing and the remaining 5650 constitute the rural community. The two populations reside in different areas and have different socioeconomic and dietary profiles. As expected, the subsistence of the fishers consists predominantly of the fish they catch, whereas the rural villagers depend more on their agricultural products. The volunteer subjects ranged in age from 20 to 60 yr. The volunteers were recruited and examined by a medical doctor at the local health center and gave informed consent to participate in this study. They all were in good general health, with no recognizable chronic disease, as stated by their clinical reports. Their alcohol, drug, and tobacco consumption was also recorded.

Blood samples were collected from May to December 1996 at the health center. It was harder to convince male volunteers, making it impossible to reach an equal or similar number of men and women recruited for the study.

\section{Blood Sampling and Storage}

Blood samples $(12 \mathrm{~mL})$ were drawn in the morning from fasting subjects following standard laboratory procedures. About $2.5 \mathrm{~mL}$ of whole 
blood were transferred into a heparinized propylene tube for determination of GSH-Px activity. The remainder was centrifuged at $1.8 \mathrm{~g}$ at $4^{\circ} \mathrm{C}$ for $15 \mathrm{~min}$ to obtain serum. A first aliquot was immediately frozen with liquid nitrogen and kept at $-196^{\circ} \mathrm{C}$ until needed for TBARS analysis. A second portion was stored at $-20^{\circ} \mathrm{C}$ for the measurement of Se levels. A third fraction was used to evaluate the lipid parameters.

\section{Assays}

\section{Serum Se Levels}

Serum Se was determined by direct electrothermal atomic absorption spectrometry with Zeeman background correction (10). The accuracy of the procedure was verified by means of Seronorm, a standard reference material consisting of lyophilized human serum from Nycomed (Roskilde, Denmark) and by participation in interlaboratory comparison trials.

\section{GSH-Px}

The GSH-Px activity in whole blood was measured by the method of Paglia and Valentine (11) with cumene hydroperoxide as the substrate (Ransel Kit, Randox Laboratories, UK). Hemoglobin concentration was measured by the cyanomethaemoglobin method (Merckotest, Merck).

\section{Lipid Peroxidation}

All reagents used for the analysis were of analytical grade, purchased from Merck (Germany). Serum lipid peroxidation was assessed by colorimetric quantification of TBARS, as described by Satoh (12), with some modifications. To optimize the extraction of the chromogen, the $\mathrm{pH}$ was adjusted to 1.6-1.7 after the reaction and before the extraction step (13). For optimal chromogen extraction, we used a $1: 15$ mixture of $n$-butanol/pyridine instead of the same volume of $n$-butanol. A calibration curve was constructed for each run by using malondialdehyde as a standard.

\section{Serum Lipids}

The high-density lipoproteins (HDLs) were obtained by adding polyethylene glycol to fresh serum samples in order to precipitate all other lipoproteins (14). Serum total cholesterol and HDL cholesterol were measured enzymatically by the cholesterol CHOD-PAP method by means of a kit from Boheringer (Mannheim, Germany). The amount of LDL cholesterol was determined by the Friedewald formula:

LDL cholesterol $=$ Total cholesterol $-(1 / 5)$ triglycerides - HDL cholesterol

Serum triglycerides were determined by an enzymatic procedure based on the triglyceride GPO-PAP method using a kit from the same manufacturer. 


\section{Statistical Analyses}

The normality of the distributions was evaluated by the Shapiro test. Group means comparisons were tested for significance by the Student's $t$ test. A variance analysis (ANOVA) was performed in order to compare age, sex, alcohol, and tobacco consumption with the evaluated parameters. In $t$-tests and in Pearson's correlation, the level of statistical significance was set at $p<0.05$. All statistical analyses were performed using Excel 97 (Microsoft Corporation, USA) and STATISTICA (Statsoft Inc., Tulsa, USA) computer software packages.

\section{RESULTS}

\section{GSH-Px Activities and Se and TBARS Concentrations}

No significant differences in whole-blood GSH-Px activity were found either in intrapopulation or interpopulation analysis, in all age groups (see Table 1). There was a tendency toward higher GSH-Px activity in the fishing population women ( $f, F)$ than in men (f, M) $(p=0.07)$. The difference is not significant and could be the result of the smaller size of the male sample.

In both men and women, serum Se levels were significantly higher in the fishing than in the rural subjects (see Table 1). The average difference between the males (about 25\%) was more than twice that found between the female groups. This seems to be a major consequence of much higher values (by 39\% and 17\%, respectively) observed in the older groups (40-60 yr) of the fishing population as compared to their rural counterparts.

Sex differences in Se concentrations were found in the fishing subjects, being higher by $24 \%$ in men than in women. No significant differences were found between age groups within each population.

Mean TBARS levels were about 39\% lower in the fishing male group than in the rural subjects (see Table 1). These values did not change significantly with respect to age.

\section{Association of GSH-Px Activity and Se and TBARS Concentrations with Lipid Profile}

The subjects from each population were divided into two groups according to their cholesterol and triglycerides levels. The normal group consisted of those individuals with total cholesterol below $250 \mathrm{mg} / \mathrm{dL}$ and/or triglycerides below $200 \mathrm{mg} / \mathrm{dL}$. The hyperlipemic group had one or both parameters above those reference values.

In the fishing population, there were seven women with total cholesterol and five with triglyceride levels above normal values, one being over $300 \mathrm{mg} / \mathrm{dL}$. Only two men had high cholesterol, but both were below 300 $\mathrm{mg} / \mathrm{dL}$. In the rural population, 10 women showed hypercholesterolemia 
Table 1

Whole-Blood GSH-Px Activity and Serum Selenium (Se) and TBARS Concentrations by Sex and Age Group in Subjects from Fishing and Rural Populations of Rabo de Peixe, St. Miguel Island, The Azores' Archipelago

\begin{tabular}{|c|c|c|c|c|}
\hline \multirow{7}{*}{ GSH-Px (U/gHb) } & $\begin{array}{c}\text { Age group } \\
\text { (years) }\end{array}$ & Sex & Fishing population & Rural population \\
\hline & all age & $\mathbf{M}$ & $44 \pm 16(26)$ & $47 \pm 18(28)$ \\
\hline & & W & $51 \pm 16(50)$ & $50 \pm 15(44)$ \\
\hline & $20-39$ & $\mathbf{M}$ & $44 \pm 18(17)$ & $44 \pm 15(14)$ \\
\hline & $20-39$ & W & $51 \pm 16(31)$ & $50 \pm 13(28)$ \\
\hline & $40-60$ & $\mathbf{M}$ & $45 \pm 12(9)$ & $50 \pm 20(14)$ \\
\hline & $40-60$ & W & $51 \pm 17(19)$ & $49 \pm 18(16)$ \\
\hline \multirow{6}{*}{$\operatorname{Se}(\mu \mathrm{g} / \mathrm{L})$} & \multirow{2}{*}{ all age } & $\mathrm{M}$ & $110 \pm 25(26)^{\mathrm{ac}}$ & $88 \pm 22(28)^{c}$ \\
\hline & & $\mathrm{W}$ & $89 \pm 20(50)^{\mathrm{ab}}$ & $80 \pm 17(44)^{b}$ \\
\hline & $20-39$ & $\mathbf{M}$ & $107 \pm 23(17)^{A}$ & $91 \pm 26(14)$ \\
\hline & $20-39$ & W & $88 \pm 21(31)^{A}$ & $81 \pm 18(28)$ \\
\hline & $40-60$ & $\mathrm{M}$ & $117 \pm 29(9)^{B C}$ & $84 \pm 19(14)^{C}$ \\
\hline & $40-60$ & W & $91 \pm 18(19)^{\mathrm{BD}}$ & $78 \pm 16(16)^{D}$ \\
\hline \multirow{6}{*}{ TBARS $(\mathrm{nmol} / \mathrm{ml})$} & \multirow{2}{*}{ all age } & $\mathrm{M}$ & $1.4 \pm 0.8(26)^{d}$ & $2.3 \pm 1.3(28)^{d}$ \\
\hline & & $\mathrm{W}$ & $1.6 \pm 1.0(50)$ & $1.9 \pm 1.4(44)$ \\
\hline & $20-39$ & M & $1.4 \pm 0.9(17)$ & $2.4 \pm 1.4(14)$ \\
\hline & $20-39$ & W & $1.9 \pm 1.2(31)$ & $2.1 \pm 1.6(28)$ \\
\hline & $40-60$ & $\mathbf{M}$ & $1.3 \pm 0.8(9)$ & $2.2 \pm 1.3(14)$ \\
\hline & $40-60$ & $\mathrm{~W}$ & $1.2 \pm 0.6(19)$ & $2.2 \pm 1.3(14)$ \\
\hline
\end{tabular}

Note: Values are mean $\pm \mathrm{SD}$. Figures in parenthesis are the number of men $(\mathrm{M})$ or women $(\mathrm{W})$.

Values sharing a common superscript letter are significantly different.

c $p<0.001$.

$\mathrm{a}, \mathrm{A}, \mathrm{B}, \mathrm{C} p<0.01$.

$\mathrm{b}, \mathrm{d}, \mathrm{D} p<0.05$.

and 6 had triglyceride contents above normal values (one $>300 \mathrm{mg} / \mathrm{dL}$ ). Among men, two showed high cholesterol levels (one $>300 \mathrm{mg} / \mathrm{dL}$ ) and four exhibited high triglyceride contents (two $>300 \mathrm{mg} / \mathrm{dL}$ ). In addition, the levels of HDL and LDL cholesterol were within the normal ranges, as related to the total cholesterol for both populations in either women or men. There were no significant differences in the lipid concentrations between the two populations (see Table 2).

The GSH-Px activity was not related to lipid profile, at least in women, in both populations. The small number of hyperlipemic men did not allow us to do a reliable comparative analysis. 


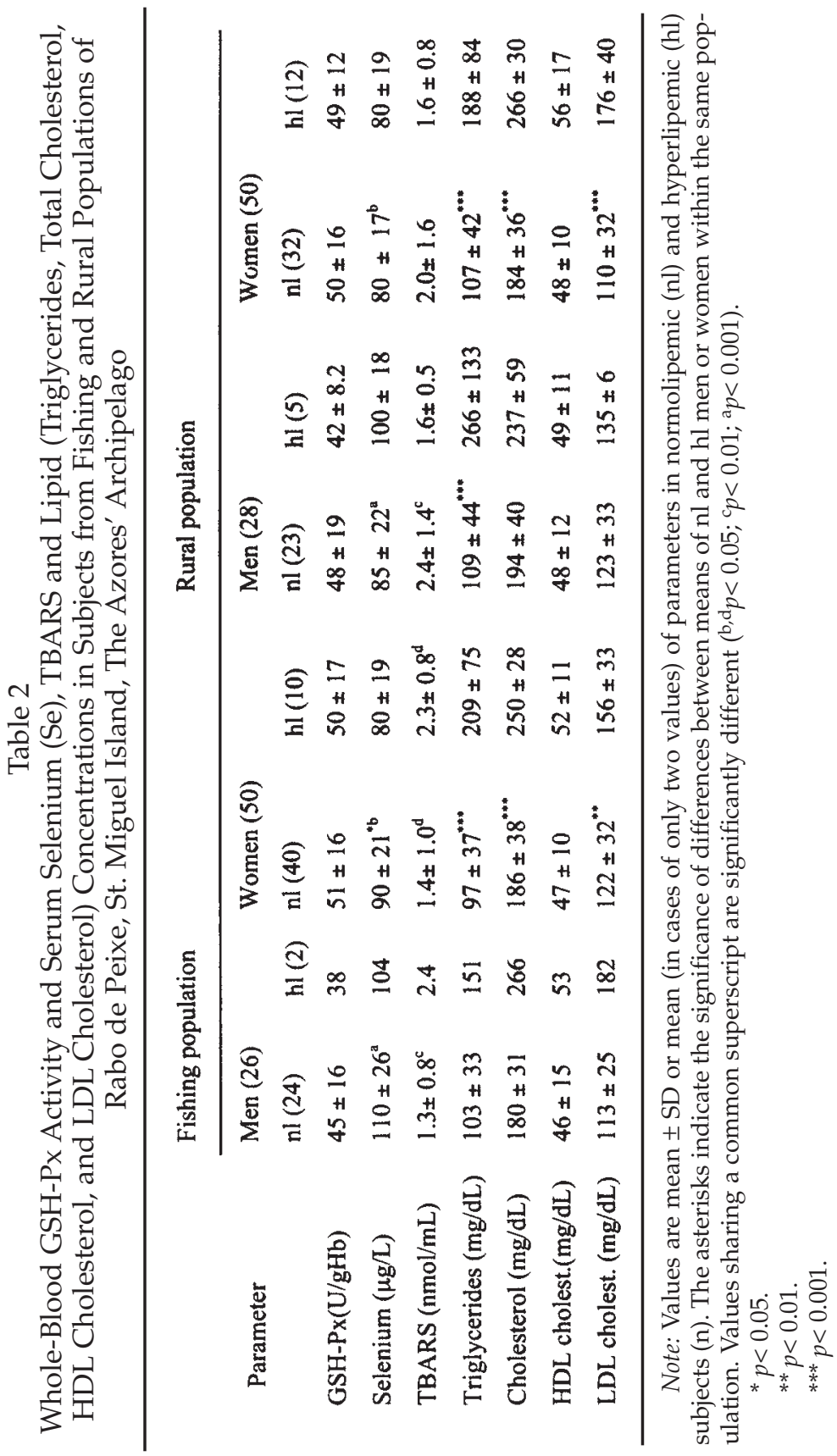


A significantly lower Se level (about 11\%) and higher TBARS (by 60\%) was observed in the hyperlipemic women (mostly with high cholesterol levels) from the fishing population as related to the controls. No such differences were noted between the rural female groups.

The TBARS mean concentrations in the fishermen with normal lipid profile were lower by $46 \%$ than that in the corresponding rural male group.

\section{Association of GSH-Px Activity and Serum Se and TBARS Concentrations with Life Habits}

There was no evidence of alcohol use among women in both populations and only two in the rural population were cigaret smokers. In the fishing population, a total of eight subjects were reported to drink alcohol on a regular basis, in a moderate manner. In the rural population, seven subjects had similar drinking profiles. Nineteen fishermen and 14 rural men were moderate smokers. Inclusion of the life habits in the statistical analysis did not show any differences in GSH-Px activity, Se levels, or TBARS in men (data not shown).

\section{Statistical Correlations}

\section{Serum Lipids and Age}

An age-related increase in triglycerides was observed in the younger subjects, but not in middle-aged subjects, in the rural population and also in fishermen (see Table 3), regardless of gender. Conversely, a positive correlation between total cholesterol and age was only found in the middleaged women of the fishing population. The same trend was observed with respect to LDL cholesterol and age, in both populations.

\section{GSH-Px, Age, and Serum Lipids}

In both populations, an increase in GSH-Px activity with age was observed in the younger male subgroups $(r=0.6, p<0.05)$. On the other hand, GSH-Px activity and HDL cholesterol correlated negatively in the middle-aged fishing population when both sexes were considered together $(n=28, r=-0.5, p<0.006)$, but not when they were taken separately.

\section{Se, Age, and Serum Lipids}

An increase of serum Se concentration with age was observed in the older rural women $(r=0.5, p<0.05)$. A weak but significant correlation was found between Se levels and HDL cholesterol in the rural population when both sexes were taken together $(r=0.24, p<0.04)$. The correlation is stronger in the younger women in this group $(r=0.4, p<0.05)$. A similar stronger correlation was found in the younger fishermen $(r=0.6, p<0.05)$. 


\section{Table 3}

Significant Correlations Found Between Some Lipid Parameters and Age in Subjects from Fishing and Rural Populations of Rabo de Peixe, St. Miguel Island, The Azores' Archipelago

\begin{tabular}{|c|c|c|c|c|c|c|c|}
\hline \multicolumn{4}{|c|}{ Fishing population } & \multicolumn{4}{|c|}{ Rural population } \\
\hline \multicolumn{4}{|c|}{ Age group (years) } & \multicolumn{4}{|c|}{ Age group (years) } \\
\hline $20-39$ & $40-60$ & & & $20-39$ & & & $40-60$ \\
\hline$M(17)$ & $\mathrm{M}+\mathrm{W}(28)$ & W (19) & & $M+W(42)$ & W (28) & M (14) & W (16) \\
\hline \multirow[t]{3}{*}{$0.56^{*}$} & & & Triglycerides & $0.55^{* * *}$ & $0.60^{* *}$ & $0.57^{*}$ & \\
\hline & $0.49^{* *}$ & $0.58^{* *}$ & Cholesterol & & & & \\
\hline & $0.48^{* *}$ & $0.61^{* *}$ & LDL-cholest. & & & & $0.53^{*}$ \\
\hline
\end{tabular}

Note: Values represent the Pearson correlation factors among triglycerides, cholesterol or LDL cholesterol, and age group of men (M) and women (W) either separated or not.

${ }^{*} p<0.05 ;{ }^{* *} p \leq 0.01 ;{ }^{* * *} p<0.001$.

\section{TBARS and Serum Lipids}

Serum TBARS and total cholesterol correlated positively in the rural population when considering men and women together $(r=0.33, p<0.01)$, in particular in the younger ones $(n=42, r=0.4, p<0.01)$. A similar correlation was observed in the whole female population $(r=0.4, p<0.05)$ and in both age groups $(r=0.45, p<0.02$ and $r=0.51, p<0.05$, respectively). Also, serum TBARS and triglyceride concentrations correlated well when taking the younger men and women in the fishing population together $(n=37, r=$ $0.5, p<0.001)$. This correlation seemed to arise mainly from the female group $(r=0.6, p<0.001)$.

\section{DISCUSSION}

These are the very first data to be reported on blood GSH-Px activity and serum Se and TBARS concentrations in rural and fishing populations from The Azores, with the particularity of being two neighboring communities within the same area that live apart from each other, with specific life habits, including diet.

\section{GSH-Px and Se}

The average whole-blood GSH-Px activities and serum Se concentrations point to an adequate nutritional Se status in both populations studied. The enzyme activity was the same for both groups, but serum Se levels were much higher in the fishing than in the rural populations. In addition, 
serum Se concentration in these fishermen was similar to the mean value $(104 \pm 21 \mu \mathrm{g} / \mathrm{L})$ found in a fishermen population from Madeira Island (15) and higher than those we have observed in two other Portuguese urban populations: Lisbon (on the mainland, $87 \pm 20 \mu \mathrm{g} / \mathrm{L}$ in women, $100 \pm 11$ $\mu \mathrm{g} / \mathrm{L}$ in men) and Ponta Delgada (located $15 \mathrm{~km}$ from Rabo de Peixe, $86 \pm$ $15 \mu \mathrm{g} / \mathrm{L}$ in women, $98 \pm 16 \mu \mathrm{g} / \mathrm{L}$ in men) $(16,17)$. In turn, Se mean values in the rural subjects from Rabo de Peixe were in the same range of those we found in another rural population in the Portuguese mainland, but significantly higher than the observed in the rural male population of Curral das Freiras, in Madeira (60 $\pm 16 \mu \mathrm{g} / \mathrm{L})(15)$. These results are probably related to the high protein and selenium content in the diet of the fishing populations (18), as compared to the rural or urban populations.

Serum Se concentrations were much higher in men than in women from the fishing community of Rabo de Peixe. A similar significant sexrelated difference had already been observed in the urban populations of Lisbon and Ponta Delgada. However, a difference based on sex has not been generally described in populations of other European countries (19).

These discrepancies in literature may be related not only to the different hormonal status of men and women but also to other aspects such as particular differences in dietary habits between the two genders. In the present fishing community, the second factor might be of some relevance, because in that group, protein intake and alcohol consumption are much more important in the men's than in women's diet.

The increase of serum Se levels with age found in the middle-aged rural women is in agreement with the results from Ponta Delgada (20) and with those reported by other authors for the same age range $(2,21)$.

There are some studies reporting a significantly higher GSH-Px activity in women when compared to men (22-24) but not in others (25). These discrepancies may be the result of differences in age ranges, physiological factors, and lifestyle. In the present work, most of the women from the fishing population seemed to have higher GSH-Px activities than men, who exhibited, however, higher serum Se levels. A sexdependent GSH-Px activity has been well known in rat liver (26). In addition, the hypothesis of a different cellular response to Se intakes including both moderate and high values in the expression of the GSHPx isozymes or other selenoproteins would stand for the regulation role of Se over selenoprotein biosynthesis claimed by many authors (27). In fact, this trace-element-dependent biosynthesis is recognized to follow a strict hierarchy in cases of limited Se supply (28). In particular, GSH-Px showed different expressions in response to Se status (29). Also, different responses to various Se intakes (low, medium, and high) of GSH-Px activities in human erythrocytes, platelets, and plasma have been reported recently (30).

An age-related impairment in oxidative defense mechanisms is well established and supports the free-radical origin of aging (31). However, erythrocyte GSH-Px activity clearly is not an age-dependent parameter. In 
fact, some authors found a positive GSH-Px-age correlation (24), whereas others did not find any correlation (32). An age-dependent elevation of erythrocyte GSH-Px in mice has recently been reported (33), but a significant reduction in the enzyme activity with age was noted in activated human neutrophils (34). In the present study, and for both communities, an increase of whole-blood GSH-Px with age was noted in younger men (which represented at least $50 \%$ of the respective population) but not in any female group. Considering that cGPx is an "emergency enzyme" to fight oxidative stress (29), our results suggest a higher stress response in young men compared to young women. The origin of this enhanced response still needs to be determined.

\section{Lipid Profile, TBARS, and Se}

A hyperlipemic condition is considered to be a risk factor for the development of atherosclerosis and vascular diseases. Dietary trace elements, including Se, contribute to reduce that risk $(9,35)$. It is also well known that aging is associated with increased serum triglyceride, total and LDL cholesterol, and reduced HDL cholesterol levels. Our results are in agreement with these statements. We emphasize the higher incidence of hyperlipemia in the rural population as compared to the fishers, where serum Se and TBARS exhibited the highest and the lowest values, respectively.

When comparing the lipid profile of subjects from Rabo de Peixe and Ponta Delgada (17), we found very significant higher triglyceride contents in both the fishing and the rural female groups, as compared to those in the urban women (26\% and 39\%, respectively). Also, HDL cholesterol mean values in women from the two populations of Rabo de Peixe were significantly lower (19\% and $17 \%$, respectively) than that in the urban group. This probably reflects different dietary habits, namely the consumption of animal fats, which are an important component in the diet of the nonurban Azorean populations.

The reason for a decreased Se concentration in the hyperlipemic women from the fishing population (but not in the rural one) is not clear. In fact, it does not agree with our observations in the population of Lisbon (20), where an increase of Se levels was found in hyperlipemic subjects. We did not find any other reference in literature. No conclusions were possible concerning the male gender because of the small number of hyperlipemic men.

The positive correlation observed between serum Se and HDL cholesterol levels in some groups from Rabo de Peixe is in accordance with results presented by Salonen et al. (36) for Eastern Finnish men. However, it disagrees with those found for healthy Dutch subjects (37) and even with those found in normolipemic Portuguese subjects (16).

We did not find any relationships of the evaluated parameters with alcohol and tobacco consumption, which, indeed, were not excessive in 
these subjects. Concerning Se, this agrees with data from other Portuguese populations $(17,20)$.

Finally, the present work points to a better oxidant/antioxidant balance in the fishing population as compared to the rural one, mainly in men, because of differences in dietary habits either between populations or genders.

Other antioxidant parameters like serum copper and zinc levels have also been determined in these communities (38), but a more detailed study taking into account age classes and lipid profile in these and in other Azorean populations will be reported later.

\section{REFERENCES}

1. D. Behne, C. Weiss-Nowak, H. Gessner, and A. Kyriakopoulos, New mammalian selenoproteins, in Trace Elements in Medicine, Health and Atherosclerosis, M. F. Reis, J. M. Pereira Miguel, AAS.C. Machado, and M. Abdulla, eds., Smith-Gordon, Nishimura, pp. 91-96 (1995).

2. H. Robberecht and H. Deelstra, Factors influencing blood selenium concentration values: a literature review, J. Trace Elements Electrolytes Health Dis. 8, 129-143 (1994).

3. K. R. Maddipati and L. J. Marnett, Characterization of the major hydroperoxide-reducing activity of human plasma, J. Biol. Chem. 262(36), 17,398-17,403 (1987).

4. M. Björnstedt, J. Xue, W. Huang, B. Akesson, and A. Holmgren, The thioredoxin and glutaredoxinsystems are efficient electron donors to human plasma glutathione peroxidase, J. Biol. Chem. 269, 29,382-29,384 (1994).

5. R. J. Kulmacz and W. E. M. Lands, Characteristics of prostaglandin II synthase, in Advances in Prostaglandin, Tromboxane, and Leukotriene Research, Volume 11, B. Samuelsson, R. Paoletti, and R. Ramwell, eds., Raven, New York, pp. 93-95 (1983)

6. H. Imai, H. Kashiwazaki, T. Suzuki, et al., Selenium levels and glutathione peroxidase activities in blood in an Andean high-altitude population, J. Nutr. Sci. Vitaminol. 41, 349-361 (1995).

7. B. P. Yu, Cellular defenses against damage from reactive oxygen species, Physiol. Rev. 74(1), 139-162 (1994).

8. H. Wiseman and B. Halliwell, Damage to DNA by reactive oxygen and nitrogen species: role in inflammatory disease and progression to cancer, Biochem. J. 313, 17-29 (1996).

9. J. Nève, Selenium as a risk factor for cardiovascular diseases, J. Cardiovasc. Risk 3, 42-46 (1996).

10. J. Nève, S. Chamart, and L. Molle, Optimization of a direct procedure for determination of selenium in plasma and erythrocytes using Zeeman effect atomic absorption spectroscopy, in Trace Element Analytical Chemistry in Medicine and Biology, Volume 4, P. Bratter and P. Schramel, eds., Walter de Gruyter, New York, pp. 349-358 (1987).

11. D. E. Paglia and W. N. Valentine, Studies on the quantitative and qualitative characterization of erythrocyte glutathione peroxidase, J. Lab. Clin. Med. 70, 158-169 (1967).

12. K. Satoh, Serum lipid peroxide in cerebrovascular disorders determined by a new colorimetric method, Clin. Chim. Acta 90, 37-43 (1978).

13. W. Wasowicz, J. Nève, and A. Peretz, Optimized steps in fluorimetric determination of thiobarbituric acid-reactive substances in serum: importance of extraction $\mathrm{pH}$ and influence of sample preservation and storage, Clin. Chem. 39, 12, 2522-2526 (1993).

14. L. I. Guidez, G. J. Miller, M. Burstein, S. Slage, and H. A. Eder, Separation and quantification of subclasses of human plasma HDL by a simple precipitation procedure, $J$. Lipid Res. 23, 1206-1223 (1982). 
15. A. M. Viegas-Crespo, M. L. Pavão, M. L. Mira, I. Torres, M. J. Halpern, and J. Nève, Comparison of serum selenium levels in inhabitants from different portuguese regions, in Therapeutic Uses of Trace Elements: Status, Epidemiology of Trace Elements and Intervention Studies, J. Nève, P. Chappuis, and M. Lamand, eds., Plenum, New York, pp. 351-354 (1996).

16. A. M. Viegas-Crespo, J. Nève, M. L. Monteiro, M. F. Amorim, O. Paulo, and M. J. Halpern, Selenium and lipid parameters in plasma of portuguese subjects, J. Trace Elements Electrolytes Health Dis. 8, 119-122 (1994).

17. A. M. Viegas-Crespo, M. L. Pavão, O. Paulo, V. Santos, M. C. Santos, and J. Nève, Trace element status (Se, $\mathrm{Cu}, \mathrm{Zn}$ ) and serum lipid profile in portuguese subjects of San Miguel Island from Azores' archipelago, J. Trace Elements Med. Biol. 14, 1-5 (2000).

18. L. Hagmar, M. Persson-Moschos, B. Akesson, and A. Schutz, Plasma levels of selenium, selenoprotein $\mathrm{P}$ and glutathione peroxidase and their correlations to fish intake and serum levels of thyrotropin and thyroid hormones: a study on Latvian fish consumers, Eur. J. Nutr. 52(11), 796-800 (1998).

19. H. Robberecht, P. Hendrix, R. Van Cauwenbergh, and H. Deelstra, Actual daily dietary intake of selenium in Belgium, using duplicate portion sampling, Z. Lebensm. Unters. Forsch. 199, 251-254 (1994).

20. A. M. Viegas-Crespo, M. L. Pavão, V. Santos, et al., Selenium status and cardiovascular risk factors in populations from different portuguese regions, in Natural Antioxidants and Food Quality in Atherosclerosis and Cancer Prevention: Selenium Intake and Status of Various Populations, J. Kumpulainen and J. Salonen, eds., The Royal Society of Chemistry, Cambridge, pp. 188-194 (1996).

21. J. Versieck and R. Cornelis, Trace elements in Plasma or Serum, CRC, Boca Raton, FL. (1989).

22. L. Guemouri, Y. Artur, B. Herbeth, C. Jeandel, G. Cuny, and G. Siest, Biological variability of superoxide dismutase, glutathione peroxidase, and catalase in blood, Clin. Chem. 37(11), 1932-1937 (1991).

23. C. Berr, A. Nicole, J. Godin, et al., Selenium and oxygen-metabolizing enzymes in elderly community residents: a pilot epidemiological study, J. Am. Geriatr. Soc. 41(2), 143-148 (1993).

24. F. Girodon, D. Blache, A.-L. Monget, et al., Effect of a two-year supplementation with low doses of antioxidant vitamins and/or minerals in elderly subjects on levels of nutrients and antioxidant defense parameters, J. Am. Coll. Nutr. 16(4), 357-365 (1997).

25. I. Ceballos-Picot, J. M. Trivier, A. Nicole, P. M. Sinet, and M. Thevenin, Age-correlated modifications of copper-zinc superoxide dismutase and glutathione-related enzyme activities in human erythrocytes, Clin. Chem. 38, 66-70 (1992).

26. R. E. Pinto and W. Bartley, The effect of age and sex on glutathione reductase and glutathione peroxidase activities and on aerobic glutathione oxidation in rat liver homogenates, Biochem. J. 112, 109-114 (1969).

27. J. Köhrle, R. Brigelius-Flohé, A. Böck, R. Gärtner, O. Meyer, and L. Flohé, Selenium in biology: facts and medical perspectives, Biol. Chem. 381, 849-864 (2000).

28. L. Flohé, E. Wingender, and R. Brigelius-Flohé, Regulation of glutathione peroxidases, in Oxidative Stress and Signal Transduction, H. J. Forman and E. Cadenas, eds., Chapman \& Hall, New York, pp. 415-440 (1997).

29. R. Brigelius-Flohé, Tissue-specific functions of individual glutathione peroxidases, Free Radical Biol. Med. 27(9/10), 951-965 (1999).

30. T. Fox, C. Atherton, S. Fairweather-Tait, et al., Changes in indices of selenium status in men on low, medium, and high intakes, in Trace Elements in Man and Animals 10, A. M. Roussel, R. A. Andersen, and A. E. Favier, eds., Kluver Academic/Plenum Ps, New York, pp. 877-881 (2000).

31. D. Harman, Free radical theory of aging: history, in Free Radicals and Aging, I. Emerit and B. Chance, eds., Birkhauser, Bazel, pp. 1-10 (1992). 
32. H. R. Andersen, J. B. Nielsen, F. Nielsen, and P. Grandjean, Antioxidative enzyme activities in human erythrocytes, Clin. Chem. 43(4), 562-568 (1997).

33. H.-K. Wong, J. Riondel, and A. Favier, Biomarkers of mouse aging: Modifications of minerals on antioxidant enzymes, in Trace Elements in Man and Animals 10, A. M. Roussel, R. A. Andersen, and A. E. Favier, eds., Kluver Academic/Plenum Ps, New York, p. 448 (2000).

34. Y. Ito, O. Kajkenova, R. J. Feuers, et al., Impaired glutathione peroxidase activity accounts for the age-related accumulation of hydrogen peroxide in activated human neutrophils, J. Gerontol. A: Biol. Sci. Med. Sci. 53(3), M169-M175 (1998).

35. Y. Rayssiguier and A. Mazur, Trace elements: metabolism and oxidative modifications of lipoproteins, in Trace Elements in Man and Animals 10, A. M. Roussel, R. A. Andersen, and A. E. Favier, eds., Kluver Academic/Plenum Ps, New York, pp. 97-103 (2000).

36. J. T. Salonen, R. Salonen, K. Seppaenen, et al., Relationship of serum selenium and antioxidants to plasma lipoproteins, platelet aggregability and prevalent ischaemic heart disease in Eastern Finnish men, Atherosclerosis 70, 155-165 (1988).

37. S. G. F. Bukkens, N. de Vos, F. J. Kok, E. G. Schouten, A. M. Bruijin, and A. Holfman, Selenium status and cardiovascular risk factors in healthy Dutch subjects, J. Am. Coll. Nutr. 9(2), 128-135 (1990).

38. M. L. Pavão, V. Santos, A. Costa, et al., Selenium, copper and zinc in some Azorean populations, in New Aspects of Trace Element Research, M. Abdulla, M. Bost, S. Gamon, P. Arnaud, and G. Chazot, eds., Smith-Gordon, London, pp. 42-44 (1999). 